\title{
AMENDMENTS
}

\section{Retraction Note: The hidden cost of genetic resistance to HIV-1}

Jeremy Luban

Retraction to: Nature Medicine https://doi.org/10.1038/s41591-019-0481-8, published online 3 June 2019.

In view of the fact that the authors of "CCR5- $\triangle 32$ is deleterious in the homozygous state in humans" have retracted their report, we wish to retract our News \& Views article, which dealt with the above study and was based on the veracity of its data.

Published online: 8 October 2019

https://doi.org/10.1038/s41591-019-0636-7

\section{Retraction Note: CCR5- $\Delta 32$ is deleterious in the homozygous state in humans}

Xinzhu Wei (iD) and Rasmus Nielsen (D)

Retraction to: Nature Medicine https://doi.org/10.1038/s41591-019-0459-6, Published online 3 June 2019.

The authors are retracting this Brief Communication to correct the scientific literature. Arising from exchanges with David Reich and colleagues, they have been made aware of a genotyping calling bias in the underlying UK Biobank data from which the main results of the study were drawn. Further analyses confirmed that the central finding of the study - that homozygous CCR5- $\triangle 32$ mutation is associated with increased mortality in the UK Biobank - is a result of this technical artifact. Because the main conclusion of the paper is invalid, both authors, Xinzhu Wei and Rasmus Nielsen, wish and agree to retract the Brief Communication in its entirety. 